GA-A23994

\title{
INTERMITTENT CONVECTION IN THE BOUNDARY OF DIII-D
}

\author{
by \\ J.A. BOEDO, D.L. RUDAKOV, R.J. COLCHIN, R.A. MOYER, \\ S. KRASHENINNIKOV, D.G. WHYTE, G.R. MCKEE, M.J. SCHAFFER, \\ P.C. STANGEBY, W.P. WEST, S.L. ALLEN, and A.W. LEONARD
}




\section{DISCLAIMER}

This report was prepared as an account of work sponsored by an agency of the United States Government. Neither the United States Government nor any agency thereof, nor any of their employees, makes any warranty, express or implied, or assumes any legal liability or responsibility for the accuracy, completeness, or usefulness of any information, apparatus, product, or process disclosed, or represents that its use would not infringe privately owned rights. Reference herein to any specific commercial product, process, or service by trade name, trademark, manufacturer, or otherwise, does not necessarily constitute or imply its endorsement, recommendation, or favoring by the United States Government or any agency thereof. The views and opinions of authors expressed herein do not necessarily state or reflect those of the United States Government or any agency thereof. 


\title{
INTERMITTENT CONVECTION IN THE BOUNDARY OF DIII-D
}

\author{
by \\ J.A. BOEDO, † D.L. RUDAKOV, ${ }^{\dagger}$ R.J. COLCHIN, ${ }^{\ddagger}$ R.A. MOYER, ${ }^{\dagger}$ \\ S. KRASHENINNIKOV, ${ }^{\dagger}$ D.G. WHYTE, $†$ G.R. MCKEE, $\diamond$ M.J. SCHAFFER, \\ P.C. STANGEBY, $\triangle$ W.P. WEST, S.L. ALLEN,, and A.W. LEONARD \\ †University of California, San Diego \\ †Oak Ridge National Laboratory \\ tUniversity of Wisconsin \\ †University of Toronto Institute for Aerospace Studies \\ †Lawrence Livermore National Laboratory
}

This is a preprint of a paper presented at the 15th International Conference on Plasma Surface Interactions in Controlled Fusion Devices, May 27-31, 2002, in Gifu, Japan, and to be published in the Proceedings.

\section{Work supported by the U.S. Department of Energy}

under Contracts DE-AC03-99ER54463, DE-AC05-000R22725, W-7405-ENG-48, and Grants DE-FG03-95ER54294, DE-FG03-96ER54373

\section{GA PROJECT 30033 JUNE 2002}




\begin{abstract}
Intermittent plasma objects (IPOs) featuring higher pressure than the surrounding plasma, and responsible for $\sim 50 \%$ of the $\mathrm{E} \times \mathrm{B}_{\mathrm{T}}$ radial transport, are observed in the scrape-off layer (SOL) and edge of the DIII-D tokamak. The skewness of probe and BES intermittent data suggest IPO formation at or near the last closed flux surface (LCFS) and the existence of holeIPO pairs. The particle content of the IPOs at the LCFS is linearly dependent on the discharge density, however, when normalized to the local averaged density, it is fairly insensitive to density variations. It is also shown that the IPOs thermalize with the background plasma within $1 \mathrm{~cm}$ of the LCFS. The IPOs appear in the SOL of both L and $\mathrm{H}$ mode discharges carrying $~ 50 \%$ of the total SOL radial $\mathrm{E} \times \mathrm{B}_{\mathrm{T}}$ transport at all radii. However, the total flux and the IPO contribution, are highly reduced in $\mathrm{H}$-mode conditions due to the increased confinement.
\end{abstract}




\section{INTRODUCTION}

A magnetic divertor [1] in a tokamak provides heat and particle exhaust and shields the main plasma from impurity contamination. Heat and particles are transported from the plasma core to the edge and scrape-off layer plasma, where they are, in turn, conducted and convected to the divertor, which is optimized to reduce structural damage and impurity release. The balance between parallel and perpendicular transport results in a SOL which is thin compared to its length [1] and the observed SOL profiles are mostly exponential [2] with short $(1-3 \mathrm{~cm})$ decay lengths. However, there is ample [3-5] evidence that the SOL profiles are sometimes wider than usual and non-exponential and flat far away from the LCFS, suggesting that perpendicular transport in these conditions can be larger than expected.

A candidate for the additional edge/SOL transport, intermittency in the fluctuations corresponding to a significant number of events above the standard deviation, has been documented extensively in linear devices [6,7] stellarators [8] and tokamaks [9-11] and its statistical properties examined [12] via a probability distribution function (PDF) [13]. The essential result is that the fluctuations depart from Gaussian behavior, featuring Skewness [13] and Kurtosis [13] that correspond to the presence of a significant number of events above the standard deviation. Furthermore, it has been proven in tokamaks (JET) and stellarators [14] (ATF, W7-AS) that the statistical properties of the fluctuations are similar and near-Gaussian in the shear layer while deviating from Gaussian into the edge or the SOL [14]. The intermittent events have been characterized using conditional averaging tools in both linear devices [6,7] and tokamaks $[15,16]$ with the result that structures propagating radially are responsible for much of the radial transport. Recent work in the CASTOR tokamak [17], using conditional averaging reported intermittent coherent structures propagating radially and poloidally in the SOL. Comparative work at the devices TJ-I and TJ-IU, concluded that intermittency bears a substantial part of the transport and most importantly, that the various properties of the intermittency (power spectra, PDF moments, etc.) are self-similar in tokamaks and torsatrons. Recent work in the JET tokamak [18] focused on the power spectra of the fluctuations, demonstrating that decay as $1 / f$ was localized in the spectral region where intermittent (or bursty) transport is dominant and interpreting the result as an indication of closeness to instability thresholds. Therefore, the universality of intermittency and the concomitant fast convective transport has been long established $[6,8,19,20]$ and there is an extensive body of work imaging the edge of various devices with fast cameras [21,22] with the result that moving plasma filaments exist in the edge/SOL. 
This paper builds on recent work [16] demonstrating the existence of intermittency in the DIII-D boundary and characterizing it. We now present results from various diagnostics strongly indicating that the origin of the intermittency phenomena is at or near the LCFS. Furthermore we present scaling of the magnitude of the density perturbations with density in L-mode and show that the IPOs quickly thermalize with the background plasma. The role of the IPOs on radial transport is a constant fraction of the total on all conditions and is quite significant at nearly $50 \%$. 


\section{EXPERIMENTAL SETUP}

The experiments were carried out on the DIII-D tokamak [23] in a variety of discharges with plasma current $\mathrm{I}_{\mathrm{p}}=1.0 \mathrm{MA}$ and toroidal field of $\mathrm{B}_{\mathrm{T}}=2.0 \mathrm{~T}$ at $\mathrm{R}=1.7 \mathrm{~m}$. For completeness, a brief survey of discharges with neutral beam heating power of up to $2 \mathrm{MW}$, featuring both low (L) and high $(\mathrm{H})$ confinement modes, with double- and singlenull divertor geometry (at the bottom of the vacuum vessel) and with standard and reversed $\mathbf{B}_{\mathrm{T}}$ directions were investigated. Otherwise identical $\mathrm{H}$ - and L-mode discharges, shown in Fig. 1, were analyzed in particular detail for comparative studies. In some discharges, the density was increased in a stepwise manner from $\left\langle\mathrm{n}_{\mathrm{e}}\right\rangle / \mathrm{n}_{\mathrm{GW}}=0.26$ to 0.5 , as shown in Figs. 1 and 3, and the probe was inserted at various times, illustrated by the vertical lines, to assure coverage at various densities.

The principal measurements were made by a fast scanning probe array [24] featuring five tips (Fig. 2 upper inset) that sense probe current $\mathrm{I}$, ion saturation current $\mathrm{I}_{\text {sat }}$, given by the expression $\mathrm{I}_{\mathrm{sat}}=1 / 2 \mathrm{~A}_{\mathrm{pr}} \mathrm{en}_{\mathrm{e}} \mathrm{c}_{\mathrm{s}}=1 / 2 \mathrm{~A}_{\mathrm{pr}} \mathrm{en}_{\mathrm{e}}$ $\left[\mathrm{k}\left(\mathrm{T}_{\mathrm{e}}+\mathrm{T}_{\mathrm{j}}\right) / \mathrm{m}_{\mathrm{i}}\right]^{1 / 2},\left(\mathrm{~T}_{\mathrm{e}}\right.$ and $\mathrm{T}_{\mathrm{i}}$ are the ion and electron temperatures respectively, $A_{p r}$ is the tip area and $\mathrm{n}_{\mathrm{e}}$ is the electron density) and floating potential, $\Phi_{f}$. These measurements were used to infer electron temperature, $\mathrm{T}_{\mathrm{e}}$, by using the harmonic method [25], the electron density, $\mathrm{n}_{\mathrm{e}}$, by using the equation above. The probe scans horizontally from the outside wall

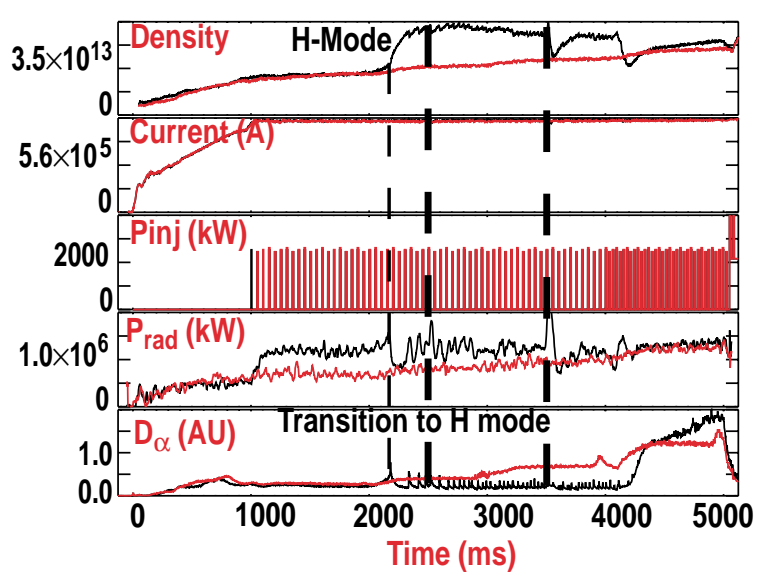

Fig. 1. Time evolution of two discharges in DIII-D showing from top to bottom, density, plasma current, injected power, radiated power and $D_{\alpha}$ signals. The transition to H-mode occurs at $2.15 \mathrm{~s}$ in one of the discharges. The probe is inserted at the times marked by the two thick vertical lines.

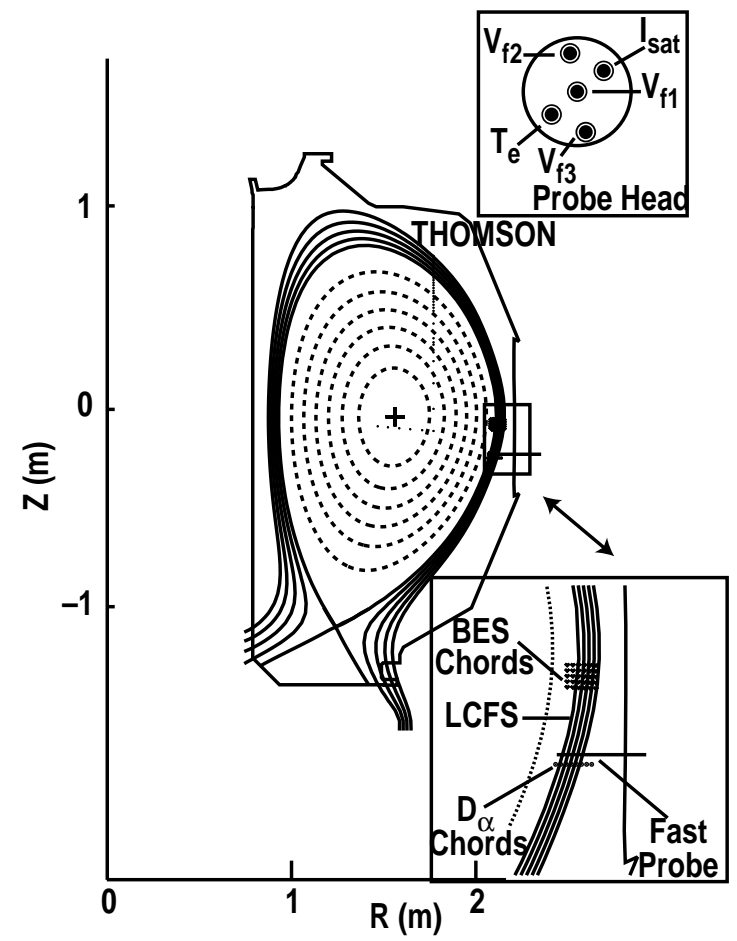

Fig. 2. Poloidal cut of DIII-D showing the magnetic geometry and some relevant diagnostics. Two insets show (top) the scanning probe tip geometry and (bottom) the BES diagnostic geometry. 
in approximately $250 \mathrm{~ms}$ along the path indicated in Fig. 2. The $\mathrm{D}_{\alpha}$ sensor array, also shown in Fig. 2, provided independent, radially-resolved intermittent signals. The beam emission spectroscopy (BES) system, configured as a $5 \times 6$ fiber array as seen in Fig. 2, was located at the edge of the plasma in the midplane to provide fast $(1 \mu \mathrm{s}) 2-\mathrm{D}$ imaging of the density. The measurements can be are mapped onto the magnetic surfaces, displayed in Fig. 2, calculated by the toroidal equilibrium fitting code EFIT [26]. 


\section{RESULTS: GENERAL DESCRIPTION OF THE INTERMITTENCY}

Appreciable intermittency is evident in many edge diagnostics [16] and the intermittent objects appear at a rate of $1-4 \times 10^{3} \mathrm{~s}^{-1}$ and, although correlation of the large excursions can be observed by eye among all the traces, conditional averaging tools, introduced in the TEXT tokamak by Filippas [15] and later in linear devices [6] and other tokamaks [8], were used to quantify the observations. A $2.5 \times$ rms-level threshold was routinely used to discriminate the intermittent events in $n_{e}, T_{e}$ and $I_{\text {sat }}$ probe data, which are then clipped for $100 \mu$ s about the maximum of each event, accumulated and then averaged. The existence of an event in a signal can be used as the condition to clip time slices in other signals at the same time slices to observe correlations.

A convenient way of visualizing the intermittency is show in Fig. 3, where data from the radial $D_{\alpha}$ array (Fig. 2) is plotted in 2-D with the ordinate corresponding to the radial direction (top is the core, bottom the SOL) and the abscissa to time. The data, which is not shown in the same scale for clarity, displays structures that appear intermittently, travel radially and poloidally and then dissipate. Notice that the structures are born in a region (marked with a dashed line) slightly inside the LCFS (marked with a solid line) only and do not come from deeper in the plasma core. The intensity of the intermittent Da signal increases linearly with density (note scale). Data from the beam emission spectroscopy (BES) diagnostic, which measures density in a 2-D array covering a $6 \mathrm{~cm} \times 5 \mathrm{~cm}$ region at the edge plasma (Fig. 2) with $1 \mu$ s resolution, was also used to visualize

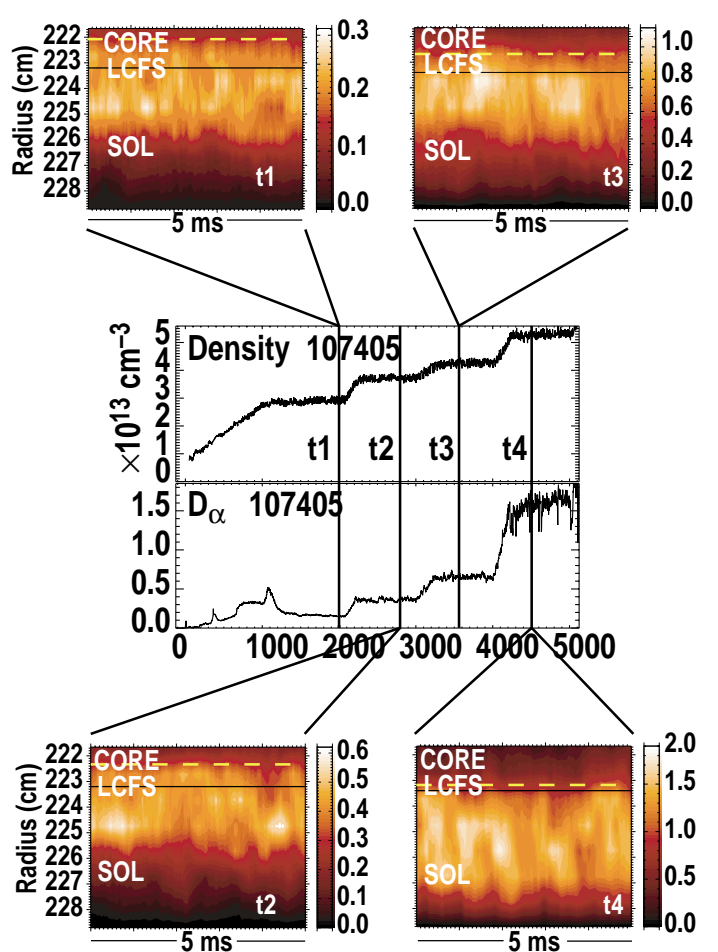

Fig. 3. The time evolution of the $L-$ mode discharge showing the density steps with $\left\langle n_{e}\right\rangle / n_{G W}$ varying from 0.27 to 0.5. Inserted 2-D plots show the time evolution of the $D_{\alpha}$ radial array signals in a $5 \mathrm{~ms}$ scale showing the intermittency and its variation with plasma averaged density. the data. Two frames taken at an arbitrary time $\mathrm{t}_{0}$ and $6 \mu$ s later $\left(\mathrm{t}_{0}+6 \mu \mathrm{s}\right)$ are shown in Fig. 4 , indicating the LCFS is indicated by a solid vertical line. The radial motion of a positive density feature, marked by a dashed circle in both frames, is indicated by vertical dashed lines. Notice that the object has a spatial extent of roughly $2 \mathrm{~cm} \times 2 \mathrm{~cm}$. From the two frames it is clear that 
the object is moving poloidally and radially with speeds that can be easily estimated at $\mathrm{V}_{\theta}=5 \mathrm{~km} / \mathrm{s}$ and $\mathrm{V}_{\mathrm{r}}=1.5 \mathrm{~km} / \mathrm{s}$ which compare well to probe data.

The intermittency causes asymmetry on statistics of the various signals introducing a deviation from Gaussian behavior, in both $\mathrm{L}-$ and $\mathrm{H}-$ mode, that is measured as skewness (i.e., positive $\mathrm{n}_{\mathrm{e}}$ bursts appear as positive skewness) [13] of $\sim 1$ in the $\mathrm{I}_{\text {sat }}$ signal at the SOL, as shown in Fig. 5(a). Similar results are obtained from BES [27,28] data, shown in Fig. 5(b). It is crucial to notice that the skewness changes sign at or near the LCFS, within the error bars of EFIT, indicating that negative events are present, corresponding to drops in the density signal from its average level.

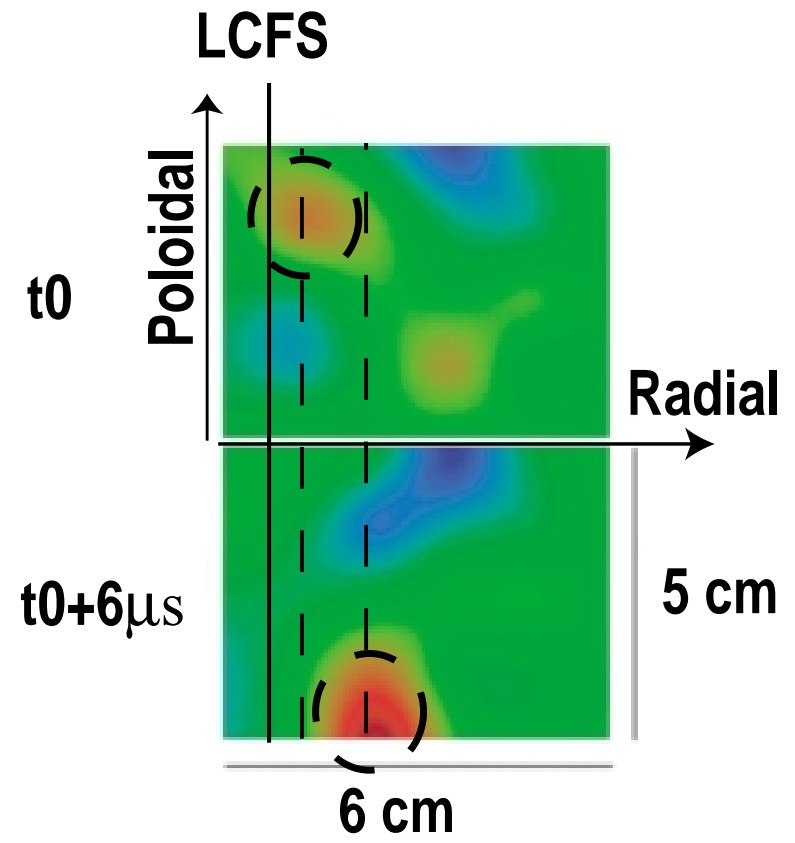

Fig. 4. Two frames from BES showing 2-D density plots. There is a time difference of $6 \mu$ s between frames. A particular structure is marked with a dashed circle, and shown in both frames, clearly highlighting poloidal and radial motion.
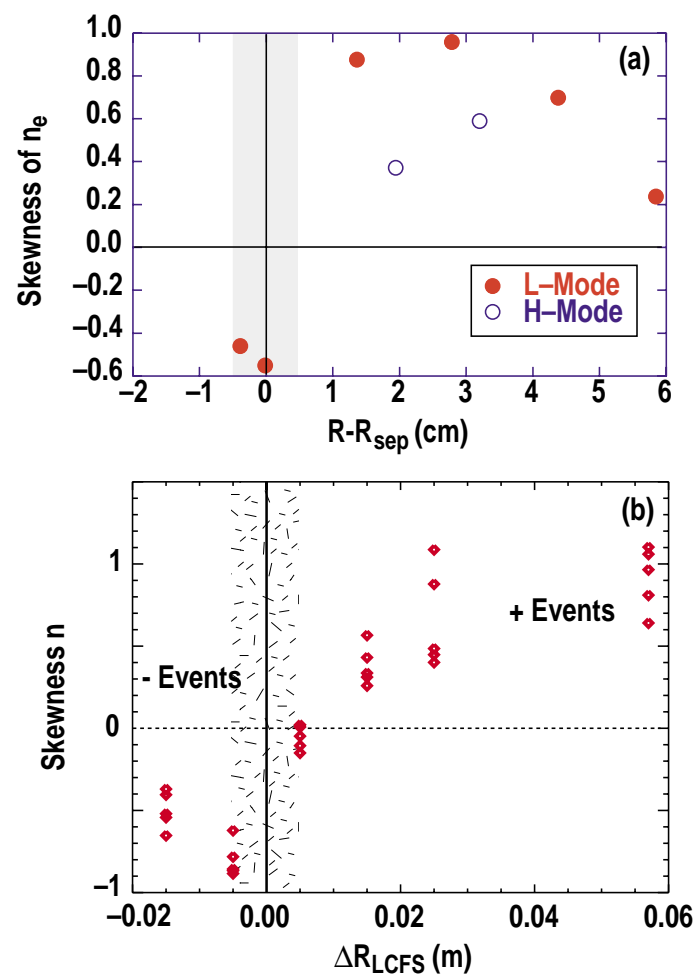

Fig. 5. The skewness of: (a) the Isat signal, and (b) the BES signal is shown for L-mode (open circles) and H-mode (solid circles) discharge conditions, showing marked deviation from Gaussian behavior. The skewnes reverses roughly at the LCFS. 
Conditional averaging performed on the probe's density data averaged over $5 \mathrm{~ms}$ (threshold at $2.5 \times$ rms level) near the LCFS, and shown in Fig. 6 for discharges with $\left\langle\mathrm{n}_{\mathrm{e}}\right\rangle / \mathrm{n}_{\mathrm{GW}}=0.26$ and 0.5 , confirm the presence of both positive and negative events. It can be seen that the positive and negative (hole) IPOs are comparable in size, suggesting that a local relaxation in the density profile is the source of the positive IPOs, and in agreement with the $D_{\alpha}$ array shown in Fig. 3.

Conditional averaging of probe signals as a function of radius produces profiles of the typical IPO, for $\mathrm{L}$ and $\mathrm{H}$-mode. The results for

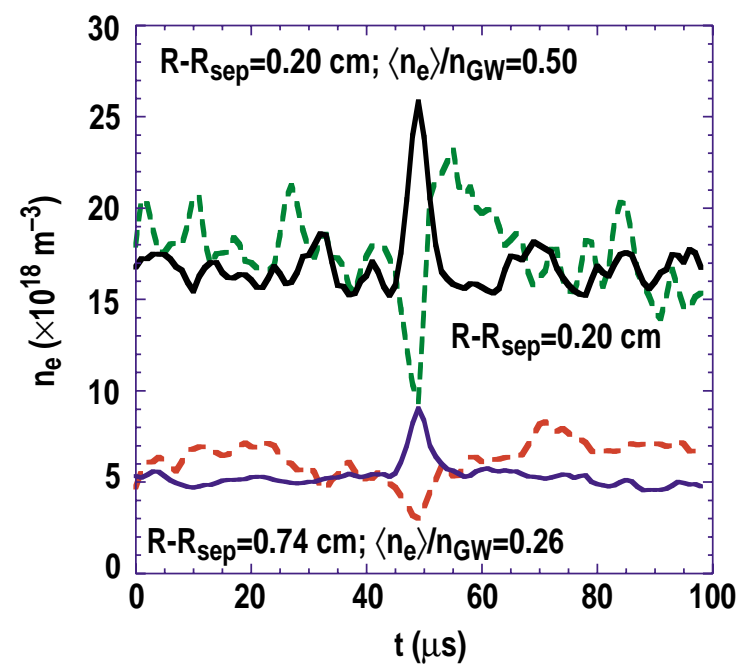

Fig. 6. Conditional averaging of the probe density signals near the LCFS for $\left\langle n_{e}\right\rangle / n_{G W}=0.27$ and 0.5 indicate the existence of density holes concomitantly with IPOs in that region. This data supports the paradigm of the IPO creation near the LCFS. density are shown in Fig. 7(a,b) for $\mathrm{L}-$ and $\mathrm{H}$-mode discharges, in the same scale for ease of comparison, by superimposing traces from various radii and indicating (arrow) the direction of increasing radius. Three properties of the data are apparent: 1) the density excursion is $2-3$ times the background density, 2) the amplitude of the pulses for similar radius is much larger for $\mathrm{L}$-mode conditions than for $\mathrm{H}$-mode conditions, and 3) the amplitude of the pulses decays rapidly with radius.

Since the interaction of the plasma with the far wall has been reported to increase with density [29], and the IPOs can provide a mechanism for fast convection of particles, it is interesting to explore the scaling of the amplitude of the density IPOs with core density. Radial profiles of the IPO peak density/temperature, and the averaged density/temperature profiles, are shown in Fig. 8(a,b) for $\mathrm{L}$-mode discharges with $\left\langle\mathrm{n}_{\mathrm{e}}>/ \mathrm{n}_{\mathrm{GW}}=0.26\right.$ and 0.5. It can be noted in Fig. 8(a) that the peak IPO density is about $70 \%$ of the background density and both decay quickly with radius with a decay length, $\mathrm{Ln}$, of $2.7 \mathrm{~cm}$. Quite different behavior is seen on the temperature, obtained from a fast $\mathrm{T}_{\mathrm{e}}$ diagnostic [30], where the IPO peak temperature decays to that of the background profile within $1 \mathrm{~cm}$. Therefore, the IPOs quickly thermalize with the background.

The relative importance of the IPO density can be evaluated as a function of $\left\langle\mathrm{n}_{\mathrm{e}}\right\rangle / \mathrm{n}_{\mathrm{GW}}$ by calculating $\left(\mathrm{n}_{\mathrm{e}}^{\text {peak }}-\left\langle\mathrm{n}_{\mathrm{e}}^{\text {aver }}\right\rangle /\left\langle\mathrm{n}_{\mathrm{e}}^{\text {aver }}\right\rangle\right)$ for various radii in the SOL, shown in Fig. 9. The outcome is quite surprising, despite the fact that the particle content (or density excursion) of the IPOs increases with density [as seen in Fig. 3 and Fig. 8(a)], it is clear that the IPOs relative 


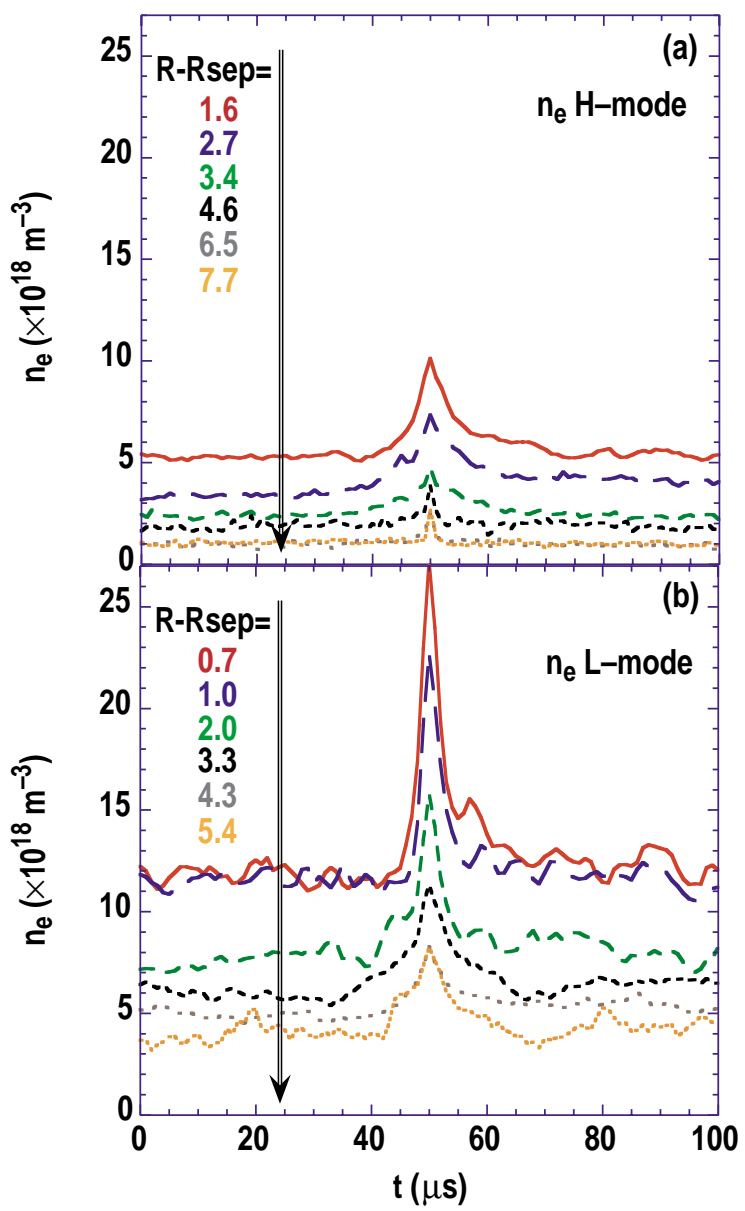

Fig. 7. Conditional averaging results showing the density pulses for $L-$ and $H$-mode conditions. Data from different radii are superimposed, those from the LCFS at the top and those from the far SOL at the bottom, as the arrows and labels indicate. Density pulses in L-mode are larger than in H-mode. Profiles are shown in Fig. 8.
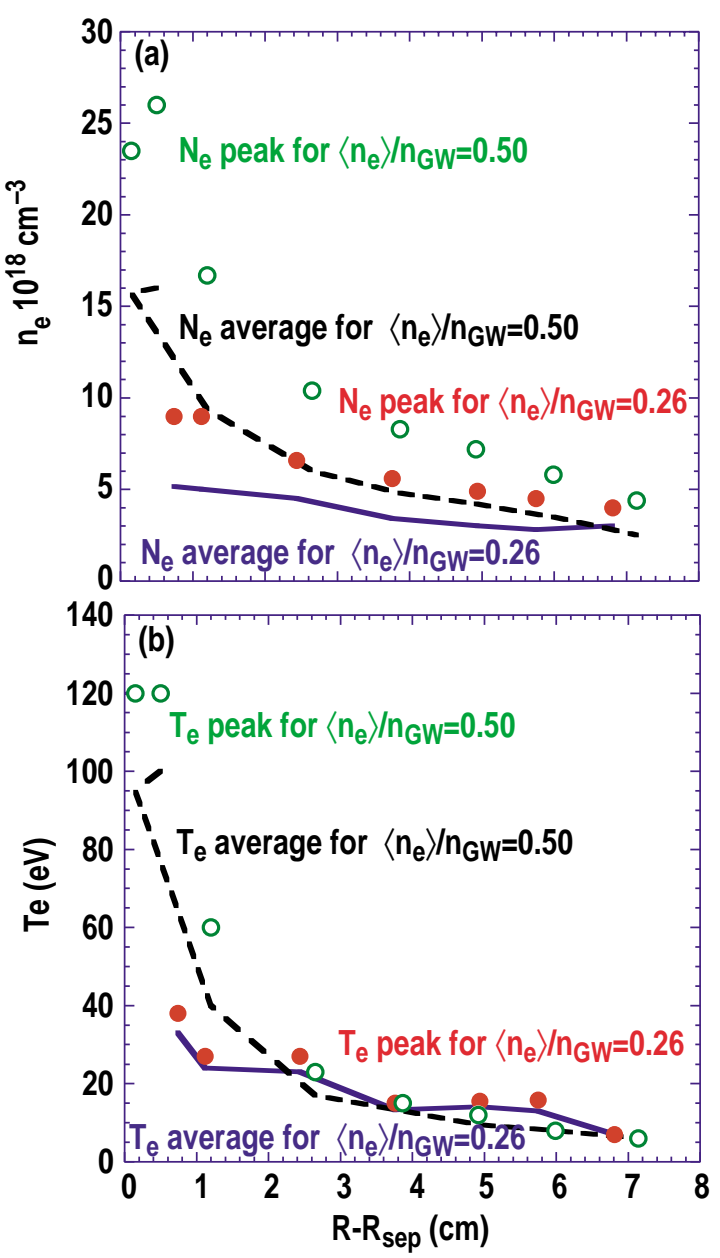

Fig. 8. Radial profiles of (a) the IPO peak density (from conditional averaging) and the average profile and $(b)$ the IPO peak temperature and the average profile for low and high density cases $\left(\left\langle n_{e}\right\rangle / n_{G W}=0.27\right.$ and 0.5$)$. The peak IPO density decays with the same scale length as the background profile while the peak temperature decays much faster.

contribution to the density is fairly constant with radius and density at about $50 \%-70 \%$. This results suggest a scaling of the IPO intensity with transport or vice versa.

Evaluation of the total particle flux convected by these events is of utmost importance to assess their relevance to the radial transport. The flux can be calculated as $\Gamma_{\text {int }}=n_{e}\left(E_{\theta} \times B / B^{2}\right)$ and the result can be seen in Fig. 10 for $\mathrm{L}-$ and $\mathrm{H}$-mode conditions and in the same scale. Three properties are again quite apparent, 1) the flux excursion is four times the background, 2) the flux for similar radius is much larger for L-mode conditions than for $\mathrm{H}$-mode conditions, and 3) the flux decays rapidly with radius. As reported earlier [16], it is found that $\Gamma_{\text {int }} / \Gamma_{\text {total }}=\left\langle\mathrm{I}_{\text {sat }} \mathrm{E}_{\theta}\right\rangle_{\text {intermittent }} /\left\langle\mathrm{I}_{\text {sat }} \mathrm{E}_{\theta}\right\rangle_{\text {total }}$, obtained in $\mathrm{L}-$ and $\mathrm{H}$-mode, is roughly $50 \%$ at all 


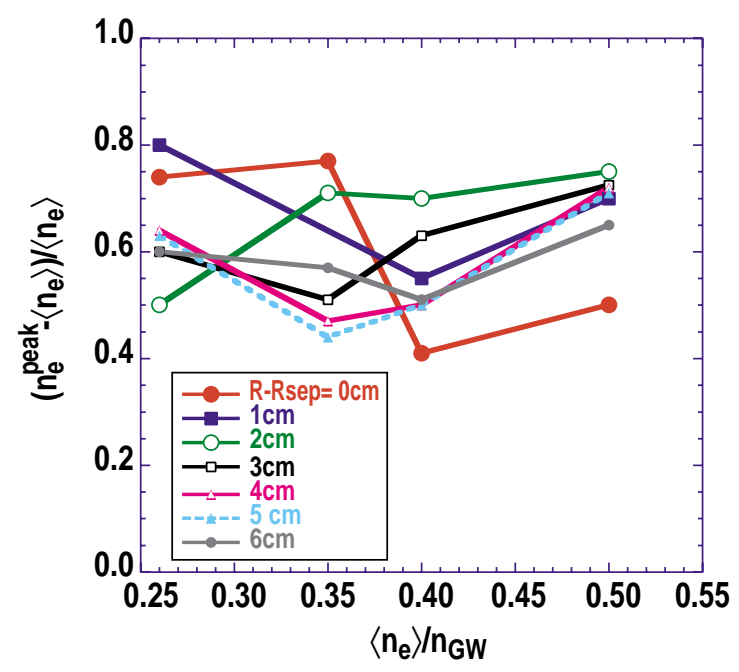

Fig. 9. The normalized intensity of the IPO density for various radius is features independence from the discharge density (in $<n_{e}>/ n_{G W}$ units).

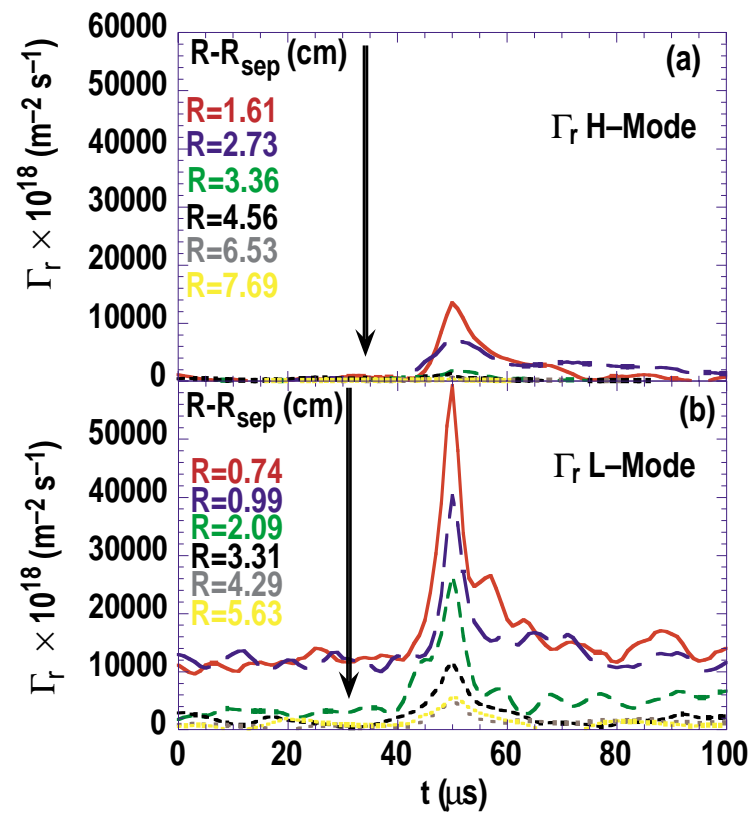

Fig. 10. Conditional averaging results showing the intermittent particle flux for $L-$ and $H$-mode conditions. Data from different radii are superimposed, those from the LCFS at the top and those from the far SOL at the bottom, as the arrows indicate. The corresponding radii are indicated. Particle flux in L-mode is larger than in H-mode.

radii. Although the relative relevance of intermittency is unchanged with radius, its absolute amplitude decays rapidly with radius. 
However, the quick reduction in the amplitude of the bursts and concomitant fast decay of the density profiles is not in contradiction with a strong particle sink in the divertor for the conditions shown. The decay of the IPO, considered as elongated structures along the magnetic field, density and temperature has been analytically predicted in recent work [31,32] to be:

$$
\mathrm{n}(\mathrm{r}, \mathrm{t})=\mathrm{n}(\mathrm{r}) /\left(1+\mathrm{t} / \tau_{\mathrm{T}}\right)^{2 \alpha / \alpha_{\mathrm{T}}} \text { and } \mathrm{T}(\mathrm{t})=\mathrm{T}_{0} /\left(1+\mathrm{t} / \tau_{\mathrm{T}}\right)^{2}
$$

where $\alpha_{\mathrm{T}}=\alpha \mathrm{S}_{\mathrm{E}} ; \tau_{\mathrm{T}}=2 /\left(\alpha_{\mathrm{T}} \mathrm{T}_{0}^{1 / 2}\right)$, $\alpha$ measures the net parallel current to the divertor plates, $\mathrm{S}_{\mathrm{E}}$ is the sheath energy transmission factor and $\mathrm{T}_{0}$ is the temperature of the IPO as it detaches from the LCFS. Since $2 \alpha / \alpha_{\mathrm{T}}=2 / \mathrm{S}_{\mathrm{E}} \ll 1$ and thus much smaller than the temperature exponent, the temperature decays much more rapidly then the density, just as observed in the experiment [Fig. 8(a) and (b)]. 


\section{CONCLUSIONS}

Intermittency in the boundary of the DIII-D tokamak during L- and H-mode discharges is present, as indicated by data from various diagnostics. The data shows that structures featuring higher density and temperature than the surrounding plasma seem to originate at or near the LCFS and travel radially in the SOL, transporting particle and energy. Although the amplitude of the IPOs in L-mode increases with density at the LCFS, they represent a fairly constant fraction $(50 \%-70 \%)$ of the local density at all radii and densities measured, Furthermore, the IPOs feature higher temperature than the background at the LCFS, but they become thermalized within a short distance of the LCFS, in agreement with analytical predictions. 


\section{REFERENCES}

[1] P.C. Stangeby, "The plasma boundary of magnetic fusion devices", IoP (200) 46.

[2] J.G. Watkins, R.A. Moyer, et al., Rev Sci. Instrum. 68, No.1 (1997) 373-376.

[3] J.A. Boedo, D. Gray, et al. Rev. Sci. Inst. Vol. 69, No. 7, (1998) 2663.

[4] B. LaBombard, M.V. Umansky, R.L. Boivin, J.A. Goetz, et al. Nucl. Fusion, Vol. 40, No. 12 (2000) 2041.

[5] M.R. Wade, et al., Jour. Nucl. Mater., 266-269 (1999) 44-50.

[6] A.H. Nielsen, H. L. Pecsell, J. Juul Rasmussen, Phys. Plasmas, Vol. 3 No. 5 (1996) 1530.

[7] R.D. Lehmer. PhD thesis, University of California Los Angeles, UCSD-ENG-032, (1996).

[8] E. Sanchez, C. Hidalgo, C. Riccardi, J. Bleuel, B. Carreras, D.E. Newman et al., Phys. Plasmas, Vol. 7, No. 5 (2000) 1408.

[9] B.K. Joseph, R. Jha, P.K. Kaw, S.K. Mattov, et al., Phys. Plas. Vol 4 (1997) 4292.

[10] R.A. Moyer, R.D. Lehmer, T.E. Evans, et al., Plasma Phys. Control Fusion, Vol. 38, (1996) 1273-1278.

[11] R. Jha and Y. C. Saxena, Phys. Plasmas, Vol. 3, No. 8, (1996) 2979.

[12] B. Carreras, et al, Phys of Plas, 7 (2000) 3278.

[13] W. Press, S. Teukolsky, W. Vettering and B. Flannery, "Numerical Recipes in Fortran", Cambridge University Press, (1986), 606.

[14] E. Sanchez, C. Hidalgo, D. Lopez-Bruna, I. Garcia-Cortes, R. Balbin, et al., Phys. Plas. Vol. 7, No. 5 (2000), 1408-1416.

[15] A. V. Filippas, R. D. Bengtson, G. X. Li, M. Meier, CH. P. Ritz, et al, Phys. Plasma, 2 (1995) 839.

[16] J.A. Boedo, D. Rudakov, R. Moyer, et al., Phys. Plasmas 8, (2001) 4826.

[17] J. Soteckel, M. V. Heller, J. Petrzilka, et al. Phys of Plas, Vol. 6, No. 3 (1999) 846. 
[18] I. Garcia-Cortes, R. Balbin, A. Loarte, J. Bleuel, et al. Plas. Phys. Control. Fusion, Vol. 42, (2000) 389-400.

[19] G. Antar, S. Krasheninnikov, et al. Accepted by Phys. Rev. Lett. (2001).

[20] G. Antar, P. Devynck, X. Garbet and S. Luckhardt, Phys. Plas., Vol. 8, No. 5, (2001) $1612-1624$.

[21] S.J.Z. and S.S. Medley, Phys. Fluids B1, (1989), 2058.

[22] R. Maqueda, G. Wurden, S. Zweben et al., Rev. Sci. Instrum. 72, (2002) 931.

[23] J.L. Luxon, L.G. Davis, Fusion Technology 8, Part 2A, (1985) 441.

[24] J.G. Watkins, J. Salmonson, R. Moyer, et al. Rev. Sci. Inst., Vol. 63, No. 10 pt.2, (1992), 4728-30.

[25] J.A. Boedo, D. Gray, M. Schaffer, et al, Rev Sci. Inst., Vol. 70, No. 7, (1999), 2997-3006.

[26] L.L. Lao, et al., Nucl. Fusion 25, (1985) 1611.

[27] G. McKee, R. Ashley, R. Durst, R. Fonck et al., Rev. Sci. Instrum. 70 (1), (1999) 913.

[28] C. Fenzi, R.J. Fonck, M. Jakubowski, G.R. McKee, Rev. Sci. Instrum. 72 (1), (2001) 988.

[29] B. La Bombard, R. Boivin, M. Greenwald, et al., Physics of Plasmas Vol. 8, No. 5, (2001) 2107.

[30] D. Rudakov, J. Boedo, R. Moyer, et al., Rev. Sci. Inst., Vol. 72, No. 1, (2001) 453.

[31] D. D’Ippolito, J. Myra, S. Krasheninnikov, Phys. Plas., Vol. 9, No. 1 (2002) 222.

[32] S. Krasheninnikov, Phys. Lett. A 283, (2001) 368. 


\section{ACKNOWLEDGMENT}

Work supported by the U.S. Department of Energy under Grants DE-FG03-95ER54294, DEFG03-96ER54373, Contracts DE-AC03-99ER54463, DE-AC05-00OR22725, and W-7405ENG-48. Discussions with D. D'Ippolito are acknowledged. 